\title{
Public Health Surveillance and Emergency Response Assistance on a State Level through the National Toxic Substance Incidents Program
}

\author{
William C. Trachtman*, Xiaoping Nie, Syed A. Ahsan, Collette Stewart-Briley and Dianne \\ M. Dugas
}

Louisiana Department of Health and Hospitals, Baton Rouge, LA, USA

\section{Objective}

In order for there to be immediate public health involvement during hazardous chemical emergencies, the Louisiana Department of Health and Hospitals (LDHH) has recently developed and implemented emergency response guidelines for handling hazardous chemical emergencies.

\section{Introduction}

Located along the Gulf Coast, Louisiana is home to strong traditional industries, such as agriculture, petrochemicals and energy, and is also home to the largest single port in the United States (Louisiana Department of Economic Development, 2009). All of these industries use hazardous materials as part of their standard operating procedure. No matter how safe an industry is, accidents do happen, and when working with hazardous materials, these accidents can be disastrous.

The Louisiana Department of Health and Hospitals (LDHH) has recently developed and implemented emergency response guidelines for handling hazardous chemical emergencies. These guidelines were developed as an expansion of a collaborative agreement with the Centers for Disease Control and Prevention / Agency for Toxic Substances and Disease Registry (CDC / ATSDR). The cooperative agreement, known as the National Toxic Substance Incidents Program (NTSIP) collects and combines information from many resources to protect people from harm caused by spills and leaks of toxic substances and is modeled partially after CDC / ATSDR's Hazardous Substances Emergency Events Surveillance (HSEES) Program (1990-2009).

\section{Methods}

As part of the guidelines, LDHH staff receives on average 30-50 chemical event reports per day (approximately 12,000 per year) from the Louisiana State Police (LSP), the National Response Center (NRC), and the Louisiana Poison Center (LPC). These reports contain information on chemical releases and other potentially hazardous events within the state. Centralized LDHH staff review the reports and then provide the nine LDHH regions of the state with copies of all the reports specific to their region. If follow-up public health action is necessary, the program can provide an emergency response packet consisting of information about the event, the chemical(s) of concern, health effects and medical management, and map(s) of the area, which display the incident location, points of interest, hospitals, schools, day care centers, nursing homes, and current contact information. A Material Safety Data Sheet may also be sent, once all necessary information is provided. Events are monitored at local clinics and hospitals to determine the need for follow-up public health investigations. Additionally, notices are sent to specific programs within LDHH when certain criteria are met.

\section{Results}

Since implementation of the guidelines in mid-2012, LDHH has assisted in the response to approximately 40 emergency incidents and because of the guidelines, an additional 10 non-emergency events were responded to by appropriate regional LDHH staff.

\section{Conclusions}

Because of these new guidelines, these events now have immediate public health involvement that can help protect lives.

\section{Keywords}

Louisiana; NTSIP; HSEES; NRC; Toxic

*William C. Trachtman

E-mail: clay.trachtman@la.gov 